\title{
End-To-End Quality of Service Control Using Adaptive Applications*
}

\author{
Dorgham Sisalem \\ GMD Fokus \\ Hardenberg Platz 2, 10625 Berlin, Germany \\ Tel. : +493025499170 \\ Fax : +4930 25499202 \\ E-mail : sisalem@fokus.gmd.de
}

\begin{abstract}
Many distributed multimedia applications exhibit flexibility to fluctuations in the network conditions. By trading off temporal and spatial quality to available bandwidth, or manipulating the playout time of continuous media in response to variations in delay, multimedia flows can keep an acceptable QoS level at the end systems. In this study, we present a new scalable scheme for adapting the transmission rate of multimedia applications to the congestion level of the network. The scheme is based on the end to end real time transport protocol (RTP). Using the proposed scheme, we investigate the efficiency of applying adaptation in utilizing network resources, reducing losses and the scalability of the scheme as well as that of the RTP protocol. The results obtained through simulations suggest the efficiency of the scheme in utilizing the network resources and decreasing the loss rates.
\end{abstract}

\section{Keywords}

Direct adjustment algorithm, adaptive applications, RTP, RTCP, scalability.

\section{INTRODUCTION}

The vast majority of multimedia applications used currently in the Internet, such as VIC (McCanne \& Jacobson 1995), vAT (Kouvelas, Hardman \& Watson 1996) and NEVoT (Baker 1994) are based on the UDP and RTP transport protocols. However, these protocols offer no means of quality of service control and can not thereby guarantee any level of QoS. Due to the fluctuations in the network conditions, the inability of UDP or RTP to sup${ }^{*}$ This work was funded in part by the BMBF (German Ministry of Education and Research)
and the DFN (German Research Network).

Building QoS into Distributed Systems A. Campbell \& K. Nahrstedt (Eds.)

(C) 1997 IFIP. Published by Chapman \& Hall 
port QoS control renders multimedia applications, often, useless. Two main approaches were introduced in the past to overcome this problem: resource reservation (Braden, Zhang \& Berson 1995) and application control (Diot, Huitema \& Turletti 1995). Reserving enough resources for supporting a certain QoS level in advance guarantees this quality level and would be the most straight forward approach for handling the problem. However, as it is usually impossible to know the exact characteristics of a certain stream in advance one would tend to over reserve resources to guarantee the requested QoS level. This would, however, lead to under utilizing the network and would get costly if one has to pay for the reserved but unused resources. Also, reservation can only be used if all the network nodes support this scheme, which currently is not the case.

Many distributed multimedia applications exhibit flexibility to fluctuations in the network conditions (Campbell, Hutchinson \& Aurrecoechea 1995). By trading off temporal and spatial quality to available bandwidth or manipulating the playout time of continuous media in response to variations in delay, multimedia flows can keep an acceptable QoS level at the end systems without necessarily requiring any changes in the network. However, such an approach requires the exchange of state information between the source end system and the network as it is the case for the available bit rate service of ATM (Shirish 1995) or the receivers as its is the case for TCP. As these state information have to traverse at least a part of the network, the source will not be able to change its sending rate immediately after a change in the congestion state of the network. So, as a source keeps on sending data with an outdated rate until a reduction request arrives data losses can not be ruled out. However, depending on the importance of the data, the context in which the applications are used and the used codecs losing a small percentage of the data is often a small price to pay in exchange for simple network architectures and low price communication.

In this paper, we present a new adaptation scheme based on the RTP protocol. The scheme is in part similar to other schemes proposed for adapting applications to the network state, see (Busse, Deffner \& Schulzrinne 1996). In our scheme, however, we take the scalability issue into consideration and test the scheme in multicast environments.

In Section 2, we present a new scheme called the direct adjustment algorithm for adapting the transmission rate of multimedia applications to the congestion level of the network. In Section 3, we test the performance of the scheme in terms of bandwidth utilization, losses and scalability.

\section{THE DIRECT ADJUSTMENT ALGORITHM}

The direct adjustment algorithm is based upon the Real Time Transport Protocol (RTP) (Schulzrinne, Casner, Frederick \& Jacobson 1996) designed within the Internet Engineering Task Force (IETF). Note that RTP is not 
a transport protocol in the common sense. That is, it offers no reliability mechanisms, has no notion of a connection and is usually implemented as part of the application and not the operating system kernel. Instead, RTP offers the application the capability of distinguishing between different media streams and keeping track of various statistics describing the quality of the session as seen by other members of the session.

RTP sessions consist of two lower-layer data streams, namely a data stream for, say, audio or video and a stream of control packets (using the sub-protocol called RTCP).

RTP data headers allow to distinguish dynamically between different audio and video encodings and have a marker to delineate video frames and audio talk spurts.

Using the control protocol (RTCP), each session member periodically multicasts control packets to all other session members. The control packets contain information about the received and transmitted data rates, delay jitter and packet losses. The desire for up-to-date control information has to be balanced against the desire to limit control traffic to a small percentage of data traffic even with sessions consisting of several hundred members. Therefore, the control traffic is scaled with the data traffic so that it makes up a certain percentage of the data rate (usually $5 \%$ with a minimum of 5 seconds between two RTCP packets).

During a conferencing session each receiver reports in its control packets the percentage of lost data noticed since sending the last control packet. At the sender site, the RTCP packets are processed and depending on the loss values reported within the RTCP packets, the sender can increase, decrease or keep its current sending rate, see Figure 1. The decision of how to change the sending rate is taken according to the following rules:

- a source starts sending data with a pre-defined rate

- initially, the sender is the non-congested state. The sender remains in this state until a control message is received indicating loss values higher than a pre-defined threshold. During this state the sender increases its transmission rate additively with a pre-defined additive increase factor divided by the number of members participating in the session for each received control message. The number of members is determined through the RTCP protocol.

$$
\text { rate }=\text { sending rate }+\frac{\text { Additive Increase Factor }}{\text { number of members }}
$$

- when receiving a control message reporting a loss ratio higher than a sender defined loss threshold, the sending rate is reduced as follows:

rate $=$ sending rate $*(1-$ loss ratio + loss threshold $)$ 
the identity of the member reporting this loss is noted as the losing member and the reported loss value is saved as highest loss. The loss threshold is the loss value the sender is willing to accept.

- control messages received from members other than the losing member with loss values lower than the highest loss are ignored

- control messages received from members other than the losing member with loss values higher than the highest loss lead to an additional rate reduction as follows:

rate $=$ rate $*(1-$ loss ratio + highest loss $)$

highest loss is then set to this new loss value and the losing member is set to the identity of the member reporting this new loss

- control messages received from the losing member with a loss value higher than the loss threshold lead to a further rate reduction

rate $=$ rate $*(1-$ loss ratio + loss threshold $)$

after receiving a control message from the losing member with a loss value lower than the loss threshold the sender goes into the non-congested state.

Additionally, the user can specify a minimum and a maximum rate thresholds. During congestion periods the sending rate should not be decreased below the minimum threshold. During network underload periods the sending rate should not be increased above the maximum limit.

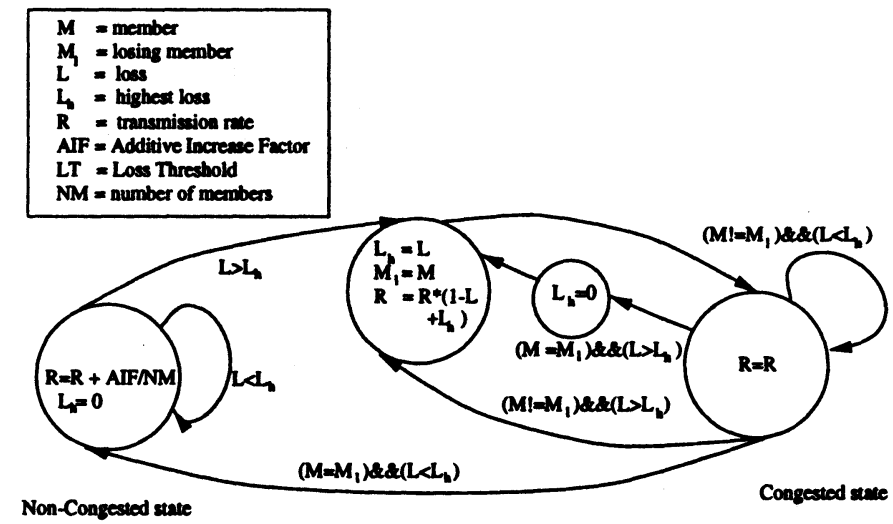

Figure 1 State diagram of the Direct Adjustment Algorithm 


\section{PERFORMANCE OF ADAPTIVE APPLICATIONS}

Through simulations we describe in this section different test cases investigating the efficiency of the direct adjustment algorithm in controlling the sending rate of an adaptive application for the case of unicast and multicast environments.

\subsection{Adaptation in the Presence of Bursty Background Traffic}

To demonstrate the behavior of senders using the direct adjustment algorithm in the event of changing traffic conditions, we simulated the case of a network consisting of a single bottlenecked link, see Fig 2.

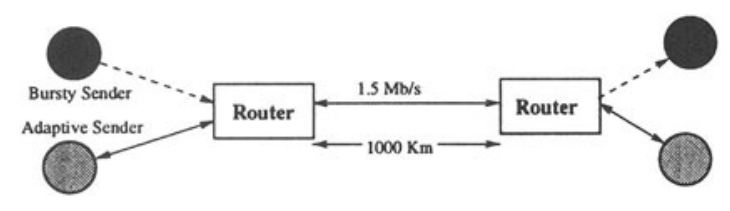

Figure 2 Test configuration for the direct adjustment algorithm

The link is shared between a bursty source and an adaptive one. The bursty source is a three state Active-Idle source as was described in (Wojnaroski 1994), see Figure 3. The number of packets generated during an active state is geometrically distributed with mean $N_{p}$. The pause between two packets during the active state is drawn from a negative exponential distribution with mean $T_{\text {off }}$. The idle states can have any general distribution with mean $T_{\text {idle }}$.

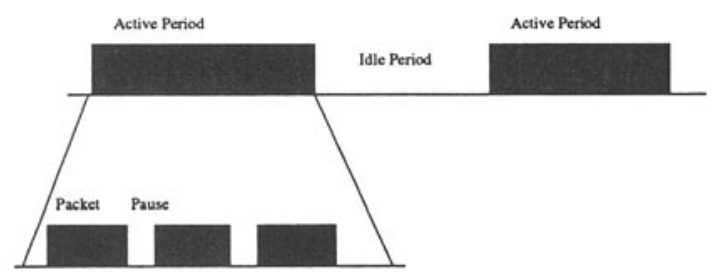

Figure 3 A three state Active-Idle source

Based on the traffic characteristics described in (Wojnaroski 1994) we tested two cases with the bursty traffic having the parameter set in Table 1. Case 1 represents typical parameters we would expect for image retrieval applications and case 2 is a representation of a distributed computing application. The 
bursty source was additionally turned on and off during the simulation to demonstrate the effects of large load changes on the algorithm.

\begin{tabular}{lccc}
\hline Test Case & $N_{p}$ & $T_{\text {off }}$ & $T_{\text {idle }}$ \\
\hline Case 1 & 500 & $2 \mathrm{msec}$. & $5 \mathrm{sec}$. \\
\hline Case 2 & 50 & $2 \mathrm{msec}$. & $0.5 \mathrm{sec}$. \\
\hline
\end{tabular}

Table 1 Parameters of the bursty traffic

The router is a random early drop (RED) gateway as was proposed by Floyd and Jacobson (Floyd \& Jacobson 1993). A RED gateway detects incipient congestion by computing the average queue size. When the average queue size exceeds a preset minimum threshold the router drops each incoming packet with some probability. Exceeding a second maximum threshold leads to the dropping of all arriving packets. This approach not only keeps the average queue length low but ensures fairness and avoids synchronization effects.

The output link of the bottleneck router has a bandwidth of $1.5 \mathrm{Mb} / \mathrm{s}$ and the end-to-end propagation delay was set to $10 \mathrm{msec}$. For the direct adjustment algorithm the initial sending bandwidth was set to $2 \mathrm{Mb} / \mathrm{s}$, the acceptable loss threshold was set to $5 \%$ and the additive increase factor to $50 \mathrm{Kbits}$. The minimum bandwidth threshold was set to $50 \mathrm{Kbits} / \mathrm{s}$ and the maximum one to $15 \mathrm{Mb} / \mathrm{s}$.

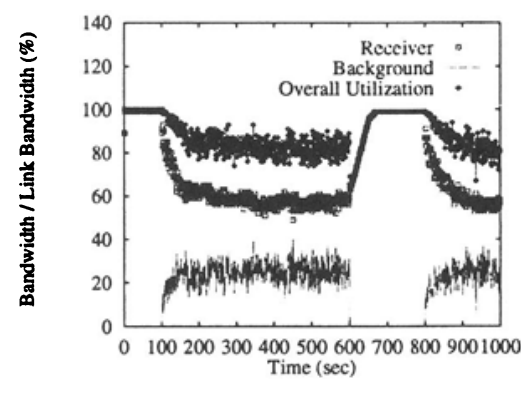

(a) Bandwidth

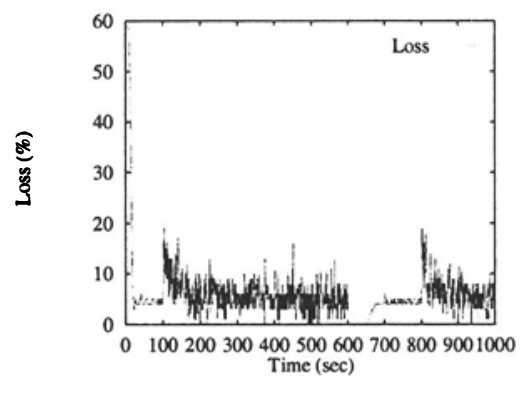

(b) Loss

Figure 4 Performance of the direct adjustment algorithm [Case 1]

Figure 4 and Figure 5 show that under changing traffic conditions the sender adapts its transmission rate in accordance with the available resources in the network. During the periods without background traffic the adaptive source 


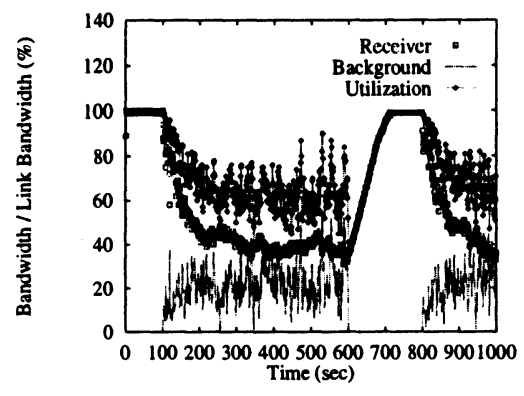

(a) Bandwidth

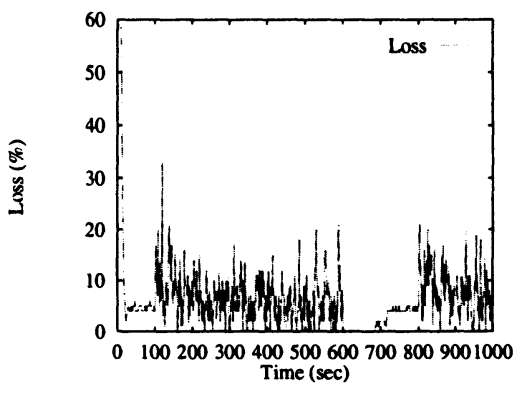

(b) Loss

Figure 5 Performance of the direct adjustment algorithm [Case 2]

increases its rate up to the link rate. The addition of the background traffic introduces losses and results in a rate reduction down to a stable level with a loss ratio of around $7 \%$ which is slightly higher than the allowed threshold of $5 \%$. Note also, that even in the presence of the very bursty traffic the overall utilization of the link remains around $80 \%$ with low loss values.

\subsection{Scalability of the Direct Adjust Algorithm}

One of the main issues when considering a QoS control approach based on feedback messages is its scalability in multicast sessions with various numbers of participants. In this section, we consider the behavior of the direct adjustment algorithm for multicast sessions with the number of participant ranging from 4 to 644 members.

The direct adjustment algorithm is based on the RTCP protocol which was designed as a scalable protocol consuming at the most $5 \%$ of the bandwidth available for a multicast session. To keep this limit the interval between two consecutive RTCP messages is set as a function of the number of the participating members. This is additionally restricted by setting the minimum interval between two messages to 5 seconds. This design implies that the number of RTCP packets sent by all members of a session should stay constant for sessions of different sizes. However, this is not completely true. As the minimum interval between two RTCP packets is limited to 5 seconds it is possible that a certain number of members does not consume all the bandwidth available for RTCP. Hence, increasing the number of members results in an increase in the number of sent RTCP packets. As an example, consider the case of a session having a session bandwidth of $1 \mathrm{Mb} / \mathrm{s}$. The RTCP share in this case would be $50 \mathrm{Kbits} / \mathrm{s}$. With only two members and an average RTCP packet length of $1 \mathrm{Kbits}$, the members would only consume $2 \mathrm{Kbits}$ 
each 5 seconds and each member would only receive 1 packet every 5 seconds. With 100 members and the average packet size of $1 \mathrm{Kbits}$, the share of each member would be $500 \mathrm{bits} / \mathrm{s}$. That is, each member would be allowed to send a packet each 2 seconds, which is still below the minimum interval of 5 seconds. Hence, as each member can at the most consume 1 Kbits each 5 seconds, the consumed RTCP bandwidth in this case is still only $20 \mathrm{Kbits} / \mathrm{s}$ and the members receive 100 packets every 5 seconds. With something like 250 members each member would get an RTCP bandwidth share of around $200 \mathrm{bits} / \mathrm{s}$ and would be allowed to send a packet each 5 seconds. Thereby, the available RTCP bandwidth would be fully consumed and the scaling mechanisms show effect with the number of members above this limit. In this case, each receiver would be getting something like 250 RTCP packets each 5 seconds. Note that the calculation presented here is only a rough approximation. The RTCP bandwidth is not distributed equally between senders and receivers. That is, senders receive a larger part of the bandwidth share dedicated to the RTCP stream. Also, with the addition of new senders the RTCP packet length increases as well. However, for the case of only one sender this calculation is acceptable. Equation 5 shows the number of members up to which the number of RTCP packets in a session increases linearly with the addition of new members and before the scaling algorithms take effect:

Number of Members $=\frac{\text { min. Interval } * \text { RTCP Bandwidth }}{\text { RTCP Packet size }}$

Algorithms reacting to every received RTCP packet such as (Busse et al. 1996) show a decreased performance when increasing the number of session members (Emanuel 1997). To account for this problem, the additive increase factor was scaled in accordance with the number of session members. This prevents the algorithm from reacting differently when increasing the number of members.

Another possible problem with the scalability of RTCP is that for large numbers of members the interval between two RTCP packets increases. As the sender can only increase its rate after receiving an RTCP packet with a loss value lower than the loss threshold, the sender will stay longer in the congested state. However, as during the congestion periods the sender does not increase its transmission rate and the sender still reacts to control messages with loss values higher than the one the sender already reacted to, no additional congestion is caused by the sender. However, the reaction to underload situations becomes slower.

As a test configuration we used one sender multicasting data through a RED-router to a different number of receivers. All receivers were connected through similar links resulting thereby in a homogeneous environment. To simplify the simulation we only considered the case of lossless RTCP transmission. That is, all RTCP messages were correctly delivered to all receivers. As background traffic we used an Active-Idle source with $N_{p}$ set to $50, T_{\text {off }}$ 
set to 0.002 seconds and $T_{\text {idle }}$ set to 5 seconds. The direct adjustment algorithm had the same parameters used in Section 3.1. That is, the acceptable loss threshold was set to $5 \%$ and the additive increase factor to $50 \mathrm{Kbits}$. The minimum bandwidth threshold was set to $50 \mathrm{Kbits} / \mathrm{s}$ and the maximum one to $15 \mathrm{Mb} / \mathrm{s}$. The link bandwidth was set to $1 \mathrm{Mb} / \mathrm{s}$ and the buffer length of the router to $10 \mathrm{KBytes}$. Those values were actually arbitrarily chosen and tests done with different parameters produced similar results.

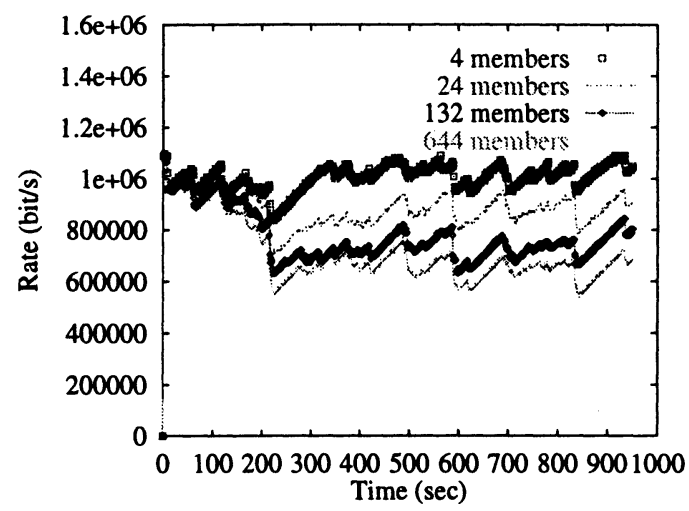

Figure 6 Bandwidth measured at the sender for multicast sessions with 4 , 24, 132 and 644 members

The results depicted in Figure 6 reveal that increasing the number of receivers reduces the average transmission rate of the sender from $1 \mathrm{Mb} / \mathrm{s}$ for the session of 4 receivers down to $700 \mathrm{Kbits} / \mathrm{s}$ for the case of 644 receivers. The performance loss is caused by the way losses are calculated and reported with RTCP. Losses are calculated as the percentage of packets that were lost divided by the number of packets that should have arrived during the time period between sending two RTCP packets. Now, consider the case of a loss peak lasting for 4 seconds, that is during 4 seconds the receivers had a loss rate of $100 \%$. If the interval between two RTCP packets was 5 seconds, starting at the beginning of the loss peak, the receiver would measure a loss rate of $80 \%$ during this measurement period. However, if the measurement period started in the middle of the loss peak, the receiver would only measure a loss rate of $40 \%$. Increasing the number of receivers, increases the probability that this situation might just happen. This results then in a rate reduction of $80 \%$ whereas the prior case would have resulted in a reduction of only $40 \%$.

Such a situation could be improved by averaging the loss values each receiver reports at the sender. This would, however, substantially increase the complexity of the scheme, as in this case the sender would have to maintain some data for each receiver, instead of just maintaining the value of the worst loss. 
We have modified the algorithm by using smoothed loss rates instead of the actual loss rates reported in the RTCP packets to determine the congestion state of the network. The smoothed loss is determined using a low pass filter as follows:

$$
\begin{aligned}
\text { smoothedLoss }_{i}= & (1-\text { smoothing factor }) * \text { smoothedLoss }_{i} \\
& + \text { smoothing factor } * \operatorname{loss}_{i}
\end{aligned}
$$

with smoothedLoss $i$ as the smoothed loss rate for member $i$ and loss $i$ as the loss value reported in the last received RTCP message from member $i$. Figure 7 depicts the results obtained by running the same simulations again using the modified algorithm and a smoothing factor of 0.5 . It is obvious that in this case increasing the number of members has no effects on the performance, as the sender keeps the same transmission level for all considered cases.

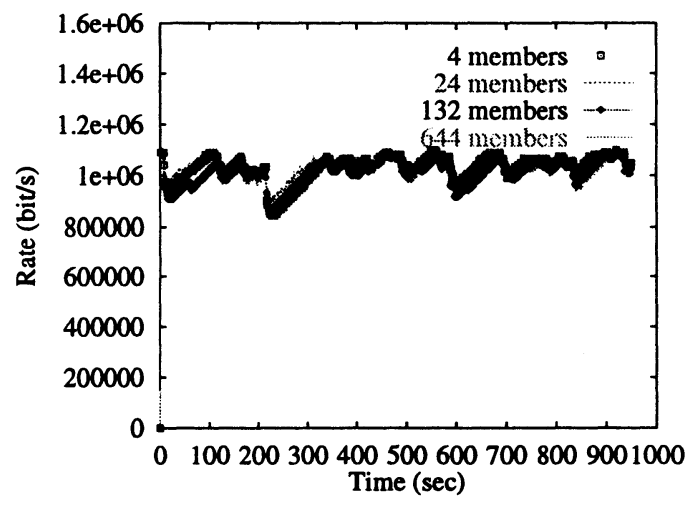

Figure 7 Bandwidth measured for multicast sessions with the modified algorithm

Another approach would be to change the way the end systems calculate and report loss values using RTCP. Instead of just reporting the loss as the average loss noticed between the sending of two consecutive RTCP messages the end systems might just report a smoothed loss rate based on the calculation we described above, see Equation 6 . This would shift the calculation complexity from the sending entities to the receiving ones, whereas each receiver would only need to calculate its own smoothed loss. This would maintain the original simplicity of the direct adjustment scheme and keep the same performance level for any number of receivers. 


\section{SUMMARY AND FUTURE WORK}

In this paper, we presented a new approach for dynamically adjusting the sending rate of applications to the congestion level observed in the network. The senders can increase their sending rate during underload situations and then reduce it during overload periods. We have run various simulations handling multicast and different background traffic patterns. The obtained results suggest the efficiency of the direct adjustment algorithm in utilizing any free bandwidth in the network while maintaining a low loss level.

However, while the adaptation scheme presented here achieves a high utilization and low loss there were some minor scalability problems that could be solved by using smoothed loss values.

In the work presented here, we did not yet address the issues of fairness and interaction with other adaptive traffic such as TCP. Some preliminary investigations that we have run suggest that adaptive schemes in general show severe fairness problems and might cause the starvation of adaptive traffic based on less tolerant reactive schemes such as TCP. These issues need to be further investigated.

Finally, investigation made on various adaptive schemes such as TCP and early ABR proposals (Sisalem \& Schulzrinne 1996) reveal that without active support from the network nodes adaptive schemes tend to be in general unfair towards long distance connections. Therefore, proposals for network services supporting adaptive end systems need to be further tested.

\section{ACKNOWLEDGMENTS}

The RTP/RTCP simulation models were mainly implemented by Timur Friedman and improved by Frank Emanuel. The comments of Henning Schulzrinne, Adam Wolisz and Frank Emanuel are gratefully acknowledged and were the basis for various aspects of this work.

\section{REFERENCES}

Baker, S. (1994), 'Multicasting for sound and video', Unix Review 12(2), 2329.

Bolot, J.-C., Turletti, T. \& Wakeman, I. (1994), Scalable feedback control for multicast video distribution in the internet, in 'SIGCOMM Symposium on Communications Architectures and Protocols', ACM, London, England, pp. 58-67.

Braden, R., Zhang, L. \& Berson, S. (1995), Resource reservation protocol (RSVP) - version 1 functional specification, Internet Draft, Internet Engineering Task Force. Work in progress.

Busse, I., Deffner, B. \& Schulzrinne, H. (1996), 'Dynamic QoS control of 
multimedia applications based on RTP', Computer Communications . *ftp://gaia.cs.umass.edu/pub/Buss9601:Dynamic.ps.gz

Campbell, A., Hutchinson, D. \& Aurrecoechea, C. (1995), Dynamic QoS management for scalable video flows, in 'Proc. International Workshop on Network and Operating System Support for Digital Audio and Video (NOSSDAV)', Lecture Notes in Computer Science, Springer, Durham, New Hampshire, pp. 107-118.

*http://spiderman.bu.edu/nossdav95/papers/campbell.ps

Diot, C., Huitema, C. \& Turletti, T. (1995), Multimedia applications should be adaptive, in 'HPCS'95', Mystic (CN).

Emanuel, F. (1997), Scalability and fairness of adaptive applications, Studienarbeit, School of Engineering, TU Berlin, Berlin. Work in progress.

Floyd, S. \& Jacobson, V. (1993), 'Random early detection gateways for congestion avoidance', IEEE/ACM Transactions on Networking 1(4), 397413.

Kouvelas, I., Hardman, V. \& Watson, A. (1996), Lip synchronization for use over the internet: Analysis and implementation, in 'GLOBECOM'96', London, UK.

McCanne, S. \& Jacobson, V. (1995), vic: A flexible framework for packet video, in 'Proc. of ACM Multimedia '95'.

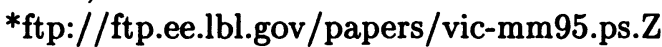

Schulzrinne, H., Casner, S., Frederick, R. \& Jacobson, V. (1996), RTP: a transport protocol for real-time applications, RFC 1889, Internet Engineering Task Force.

$*_{\mathrm{ftp}} / / /$ ds.internic.net/rfc/rfc1889.txt

Shirish, S. S. (1995), ATM Forum traffic management specification version 4.0, Technical Report 94-0013R6, ATM Forum.

Sisalem, D. \& Schulzrinne, H. (1996), Congestion control in TCP: Performance of binary congestion notification enhanced TCP compared to reno and tahoe, in 'ICNP'96', IEEE, Columbus, Ohio.

*ftp://ftp.fokus.gmd.de/pub/step/papers/Sisa9610:Congestion.ps.gz

Wojnaroski, L. (1994), Baseline text for traffic management sub-working group, Technical Report 94-0394r5, ATM Forum.

\section{BIOGRAPHY}

Dorgham Sisalem received his Master's thesis in Electrical Engineering at the Technical University Berlin in 1995. Since then he has been working as a researcher at the GMD FOKUS in Berlin. His work items include multimedia communication, QoS control and congestion control in ATM and IP-based networks. 